\title{
Visual Model BaSed Single IMAge DeHaZING USING ARTIFICIAL BEE COLONY OPTIMIZATION
}

\author{
S.MohamedMansoorRoomi ${ }^{\# 1}$ R.Bhargavi ${ }^{\# 2}$ S.Bhumesh $^{\# 3}$ \\ Department of Electronics and Communication Engineering, ThiagarajarCollege of \\ Engineering,Madurai \\ 1 smmroomi etce.edu \\ 2 rbhargavi22@gmail. com \\ 3 bhumesh.wave@gmail.com
}

\begin{abstract}
Images are often degraded by atmospheric haze, a phenomenon due to the particles in the air that scatter light. Haze induces a loss of contrast,its visual effect is blurring of distant objects. This paper presents a novel algorithm for improving the visibility of an image degraded by haze. The proposed method uses a cost function based on human visual model to estimate airlight map. It employs Artificial Bee Colony optimization $(A B C)$ as the optimization technique for estimating air light map. Image is dehazed by removing the estimatedairlight from the degraded image. The performance of the algorithm is tested and compared with various other dehazing methods and the proposed algorithm dehazes the image effectively outperforming other methods.
\end{abstract}

\section{KEYWORDS}

Airlight map,Artificial Bee Colony optimization, cost function.

\section{INTRODUCTION}

During propagation solar radiation interacts with the atmosphere, generating a variety effects upon the resulting satellite image that must subsequently be accounted for through atmospheric Corrections. Some atmospheric effects, such as cloud, block almost all radiation in the visible and infrared spectral regions. Others partly obscure the ground-reflected radiation leaving an underlying ground information component in contaminated form. "Haze" is an example of this latter effect. Haze is a commonly used term in image analysis, referring to a set of atmospheric effects that reduce image contrast. In general, the impact of haze is evident when viewing images in blue or green parts of the electromagnetic spectrum. At those wavelengths, it is generally an additive radiometric effect and varies spatially, with the resulting satellite image typically exhibiting the underlying ground cover in a diffused pattern. It is imperative that haze be removed prior to scene analysis. Several different atmospheric scattering or "haze" detection and removal techniques have been reported in the literature.In [7],assuming the scene depth is given, atmospheric effects are removed from terrain images taken by a forward-looking airborne camera. In [4] when two images are given, polarised haze effects are removed. For each photographical image, a polarising filter is attached where the image acquisition device is identically placed. The resulting images would be images which differ only in magnitude of polarised haze light

DOI : $10.5121 /$ ijist.2012.2306 
International Journal of Information Sciences and Techniques (IJIST) Vol.2, No.3, May 2012

component. In [9] estimation of several parameters are done automatically with the assumption that the higher spatial-bands of the direct transmission, the surface radiance reaching the camera, and the polarized haze contribution are uncorrelated. In [12]depending on transmission and to suppress noise amplification during dehazing , a regularisation mechanism is proposed. A user interactive tool for removing weather effects is described in [2] This method requires manually to identify regions that are heavily affected by haze or to provide some coarse depth information. In the airlight is assumed to be constant over the entire image and is estimated given a single image,based on the fact that in natural images the local sample mean of pixel intensities is proportional to the standard deviation. This work presents a novel image processing technique to estimate optimal airlight map as in Section II. Results and Conclusion are provided in Section III and Section IV.

\section{Proposed METHOD}

The first phase of this work involves estimation of optimal air light map using Artificial Bee Colony Optimization(ABC) second phase involves an effective method to correct the degraded image by subtracting the estimated air light map from the degraded image. The flowchart for proposed algorithm is shown in Figure 1.

\subsection{Haze Effect on Image}

The hazy image is degraded by airlight that is caused by scattering of light with particles in air. Airlight plays the role of being an additional source of light asmodeled in [6] and Eqn (1) below.

$$
I_{R, G, B}^{\prime}=I_{R, G, B}+\lambda_{R, G, B}
$$

where $I_{R, G, B}^{\prime}$ is the degraded image, $I_{R, G, B}$ is the original image, and $\lambda_{R, G, B}$ represents the airlight for the Red, Green and Blue channels. This relationship can be applied in the case where airlight is uniform throughout the whole image. However, the contribution of airlight is not usually uniform over the image because it is a function of the visual depth, which is the distance between the camera and the object. Therefore, the model can be modified to reflect the depth dependence as follows.

$$
I_{R, G, B}^{\prime}(d)=I_{R, G, B}(d)+\lambda_{R, G, B}(d)
$$




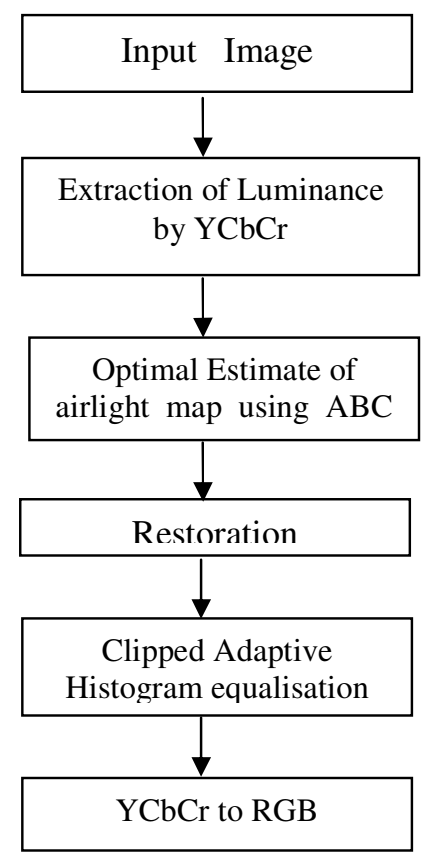

Figure 1 .Flowchart of the Proposed

Algorithm

Note that "d" represents depth. Unfortunately, it is very difficult to estimate the depth using one image taken in hazy weather conditions, so we present an airlight map that models the relationship between the coordinates of the image pixels and the airlight. In this paper, since the amount of scattering of a visible ray by large particles like haze and clouds are almost identical, the luminance component is used alone to estimate the airlight instead of estimating the R, G, and B components. The luminance image can be obtained by a fusion of the R, G, and B components. Subsequently, the color space is transformed from RGB to YCbCr. Therefore Eqn (2) can be reexpressed as follows.

$$
Y_{R, G, B}^{\prime}(d)=Y_{R, G, B}(d)+\lambda_{R, G, B}(d)
$$

where $\mathrm{Y}^{\prime}$ and $\mathrm{Y}$ reflect the degraded luminance and clear luminance images respectively at position $(i, j) . I n$ order to restore the image blurred by haze, we need to estimate the airlight map and subtract the airlight from the hazy image as follows.

$$
\hat{Y}(i, j)=Y^{\prime}(i, j)-\hat{\lambda}_{s}(i, j)
$$

In this model, $\hat{Y}$ represents the restored image and $\hat{\lambda}$ is the estimated airlight map. 


\subsection{Optimal Estimate of Airlight Using Artificial Bee Colony}

The luminance image is divided into various blocks and for each block airlight is estimated.In order to estimate the air light, we improved the cost function method in [6] using a compensation that is based on the human visual model. In Eqn (3), the airlight is to be estimated to restore the image degraded by haze. To estimate the airlight, the human visual model is employed. As described by Weber's law, a human is more insensitive to variations of luminance in bright regions than in dark regions.

$$
\Delta S=k \frac{\Delta R}{R}
$$

where $R$ is an initial stimulus, $\Delta R$ is the variation of thestimulus, and $\Delta S$ is a variation of sensation. In the hazy weather conditions, when the luminance is already high, a human is insensitive to variations in the luminance. We can estimate the existing stimulus in the image signal by the mean of the luminance within a region. The variation between this and hazy stimulus can be estimated by the standard deviation within the region. Thus the human visual model would estimate the variation of sensation as

$$
\begin{aligned}
& \frac{\operatorname{STD}(Y)}{\operatorname{mean}(Y)}=\frac{\sqrt{\frac{1}{n} \sum\left(y_{i}-\bar{Y}\right)^{2}}}{\bar{Y}} \\
& A(\lambda)=\frac{\operatorname{STD}\left(Y^{\prime}-\lambda\right)}{\operatorname{mean}\left(Y^{\prime}-\lambda\right)}
\end{aligned}
$$

In Eqn (7), increasing $\lambda$ causes an increase in $A(\lambda)$, which means that a human can perceive the variation in the luminance. However, if the absolute value of the luminance is too small, it is not only too dark, but the human visual sense also becomes insensitive to the variations in the luminance that still exist. To compensate for this, a second function is generated as follows.

$$
B^{\prime}(\lambda)=\operatorname{mean}\left(Y^{\prime}-\lambda\right)
$$

Eqn (8) indicates information about mean of luminance. In a hazy image, the result of Eqn (8) is relatively large. And, increasing $\lambda$ causes a decrease in $B(\lambda)$ which means thatoverall brightness of the image decreases.Functions (7) and (8) reflect different scales from each other. Function (8) is re-scaled to produce Eqn (9) to set 0 when input image is Ideal. Note that "Ideal" represents the ideal image having a uniform distribution from the minimum to the maximum of the luminance range. In general, the maximum value is 235 while the minimum value is 16 .

$$
B(\lambda)=\operatorname{mean}\left(Y^{\prime}\right)-\lambda * \frac{\operatorname{STD}(\text { ideal })}{\text { mean }(\text { ideal })}
$$

The $\lambda$ satisfying Eqn (10) is the estimated optimal airlight.

$$
\hat{\lambda}=\underset{\lambda}{\arg \min }\{|A(\lambda)-B(\lambda)|\}
$$

Estimation of optimal air light is performed by Artificial Bee Colony Optimization.In the ABC algorithm,the position of a food source represents a possible solution of the optimization problem and the nectar amount of a food source corresponds to the quality (fitness) of the associated 
solution. The number of the employed bees or the onlooker bees is equal to the number of solutions in the population. At the first step, the $\mathrm{ABC}$ generates a randomly distributed initial population $P(G=0)$ of $\mathrm{S}_{\mathrm{N}}$ solutions (food source positions), where $\mathrm{S}_{\mathrm{N}}$ denotes the size of population. Each solution (food source) $x_{i}\left(i=1,2, \ldots, \mathrm{S}_{\mathrm{N}}\right)$ is a $D$-dimensional vector. Here, $\mathrm{D}$ is the number of optimization parameters. The population of the positions (solutions) is subjected to repeated cycles, $C=1,2, \ldots C_{\max }$, of the search processes of the employed bees, the onlooker bees and scout bees when the initialization is completed. In the memory of onlooker bee , a position for finding a new food source and to test the nectar amount (fitness value) of the new source (new solution) is produced.If the nectar amount of the new source is higher than that of the previous one the bee remembers the new position and eliminates the previous position else the previous position is maintained. When the search processis completed by all employed bees, the nectar information of the food sources (solutions) is shared among them and their position information with the onlooker bees on the dance area. An onlooker bee evaluates the nectar information taken from all employed bees and chooses a food source with a probability related to its nectar amount. As in the case of the employed bee, she produces a modification on the position (solution) in her memory and checks the nectar amount of the candidate source (solution). Providing that its nectar is higher than that of the previous one, the bee memorizes the new position and forgets the old one.

An onlooker bee chooses a food source depending on the probability value associated with that food source, $p_{i}$, calculated by the following expression (11)

$$
p_{i=\frac{F I T_{i}}{\sum_{n=1}^{S_{N}} F I T_{n}}}
$$

where fit $\mathrm{i}_{\mathrm{is}}$ the fitness value of the solution $i$ evaluated by its employed bee, which is proportional to the nectar amount of the food source in the position $i$ and SN is the number of food sources which is equal to the number of employed bees $\left(\mathrm{B}_{\mathrm{N}}\right)$. In this way, the employed bees exchange their information with the onlookers. In order to produce a candidate food position from the old one, the $\mathrm{ABC}$ uses the following expression (12)

$$
\mathrm{v}_{\mathrm{ij}}=\mathrm{x}_{\mathrm{ij}}+\varphi_{\mathrm{ij}}\left(\mathrm{x}_{\mathrm{ij}}-\mathrm{x}_{\mathrm{kj}}\right)
$$

Where $k \in\left\{1,2, \ldots, \mathrm{B}_{\mathrm{N}}\right\}$ and $j \in\{1,2, \ldots, D\}$ are randomly chosen indexes. Although $k$ is determined randomly, it has to be different from $i . \varphi_{i, j}$ is a random number between $[-1,1]$. It controls the production of a neighbour food source position around $x_{i, j}$ and the modification represents the comparison of the neighbour food positions visually by the bee. Equation 12 shows that as the difference between the parameters of the $x_{i, j}$ and $x_{k, j}$ decreases, the perturbation on the position $x_{i, j}$ decreases, too. Thus, as the search approaches to the optimum solution in the search space, the step length is adaptively reduced.

\subsection{Smoothed Airlight Map}

When the airlight map obtained through $\mathrm{ABC}$ is directly used for restoration ,it would create blocking lines effects in the restored image. The sharp transitions among the blocks creates undesired effects.In order to resolve this issue, the airlight map is further processed.The resultant

smoothed airlight map $\hat{\lambda}_{s}$ is generated by further convolving optimal airlight map

$(\hat{\lambda})$ with Gaussian window which is used to restore haze free image. 


\subsection{Restoration}

In order to restore the luminance image, the smoothed optimal airlightmap $\left(\hat{\lambda}_{s}\right)$ is subtracted from the degraded image as Eqn (13). The term ' $b$ ' is mean value of airlight map obtained.

$$
\hat{Y}(i, j)=Y^{\prime}(i, j)-\hat{\lambda}_{s}(i, j)+b
$$

The restored luminance image $(\hat{Y})$ is further processed by clipped adaptive histogram equalization.

\subsection{Clipped Adaptive Histogram Equalization}

CLAHE addresses the issue of over amplification of noise that adaptive histogram equalisation produces by limiting the contrast enhancement. The slope of the transformation function gives the contrast amplification in the vicinity of a given pixel value. This is proportional to the slope of the neighbourhood cumulative distribution function (CDF) and therefore to the value of the histogram at that pixel value. Before computing the $\mathrm{CDF}$, amplification is limited by clipping the histogram at a predefined value thereby limiting the slope of the CDF and therefore of the transformation function. The value at which the histogram is clipped, the so-called clip limit, depends on the normalization of the histogram and thereby on the size of the neighbourhood region. CLAHE operates on small regions in the image, called tiles, rather than the entire image. Each tile's contrast is enhanced, so that the histogram of the output region approximately matches the histogram specified by the 'Distribution' parameter. The neighbouring tiles are then combined using bilinear interpolation to eliminate artificially induced boundaries. The contrast, especially in homogeneous areas, can be limited to avoid amplifying any noise that might be present in the image. By changing the colour space from $\mathrm{YCbCr}$ to $\mathrm{RGB}$, dehazed colour image can be obtained.

\section{RESUlTS AND DisCUSSION}

The proposed algorithm is tested with haze images and compared against various Dehazing algorithms. He etal proposed Dehazing based on dark channel prior [14],Ding etal proposed Colour contrast enhancement [15], Xie etal proposed haze removal based on retinex theory [16] .The proposed method is also compared with dehazing using Particle Swarm optimisation technique [17] .The effectiveness of the proposed algorithm is evaluated by the tenengrad criterion and contrast improvement index.

\subsection{Tenengrad}

In order to evaluate the effectiveness of the resultant image a well-known benchmark-image sharpness measure, the tenengrad criterion as in Eqns (14)-(15) are used. The tenengrad criterion is based on gradient,at each $\operatorname{pixel}(x, y)$, were the partial derivatives are obtained by a high-pass filter, eg., The gradient magnitude is given by: sobel operator, with the convolution kernels

$$
\begin{aligned}
& S(x, y)=\sqrt{\left(i_{x} * I(x, y)\right)^{2}+\left(i_{y} * I(x, y)\right)^{2}} \\
& T E N=\sum_{x} \sum_{y} S(x, y)^{2}, S(x, y)>T
\end{aligned}
$$

And the tenengrad criteria is formulated as were $\mathrm{T}$ is the threshold. The quality of the image is usually considered better if its tenengrad value is higher. 


\subsection{Contrast Improvement Index}

Let $\mathrm{I}$ be the given image of size NxM.For every pixel at $\mathrm{I}(\mathrm{x}, \mathrm{y})$ a block of $\mathrm{n} 1 \mathrm{x} \mathrm{n} 1$ size is taken.Each block is passed to the contrast finding function.Each block passed is further broken into small windows of size $\mathrm{n} 2 \mathrm{xn} 2$. Mean pixel intensity $\mathrm{M}(\mathrm{w})$ is calculated for each of the windows using the equation (16).

$M(w)=\frac{\sum_{x=0}^{W} \sum_{y=0}^{W} b(x, y)}{W_{x} W_{y}}$

$\mathrm{N}$-no of windows in the block

W-size of the window

Further the Mean absolute deviation $\mathrm{D}(\mathrm{w})$ is calculated for each window.

$D(w)=\frac{\sum_{x=0}^{W} \sum_{y=0}^{W} b(x, y)-M(w)}{W_{x} W_{y}}$

Using the calculated Mean pixel intensity, $\mathrm{M}(\mathrm{w})$ and Mean absolute deviation, $\mathrm{D}(\mathrm{w})$ values the Deviation of the mean pixel intensity, $D_{m}$ and the Mean of the deviation values $\mu_{d}$ are calculated.

$\mu_{d}=\frac{\sum_{y=0}^{W} D(w)}{N}$

$D_{m}=\frac{\sum_{x=0}^{W} M(w)-\sum_{y=0}^{W} \frac{D(w)}{N}}{N}$

The sum of the calculated deviation and calculated mean would represent the contrast of the nth block, $C_{n}$. Similarly the contrast of each and every block would be calculated. Contrast value of the nth block is given by Eqn (20)

$C_{n}=\mu_{d}+D_{m}$

B-total no of blocks. In this way the contrast of the image is calculated. Improved contrast measure shows the significance of the proposed method.

The tenengrad criterion is based on gradient at each pixel andthe image quality is usually considered higher if its tenengrad value is larger. Results are shown in Figure 2-4 and the corresponding tenengrad values and contrast improvement index for various images is computed and tabulated in Table.1 and Table.2. Figure 2(a) and Figure 2(b) shows the restored image by estimated airlight map of image 1 before and after smoothing respectively. Figure 3 and Figure 4 shows the dehazed images obtained by various other Dehazing algorithms. 
International Journal of Information Sciences and Techniques (IJIST) Vol.2, No.3, May 2012

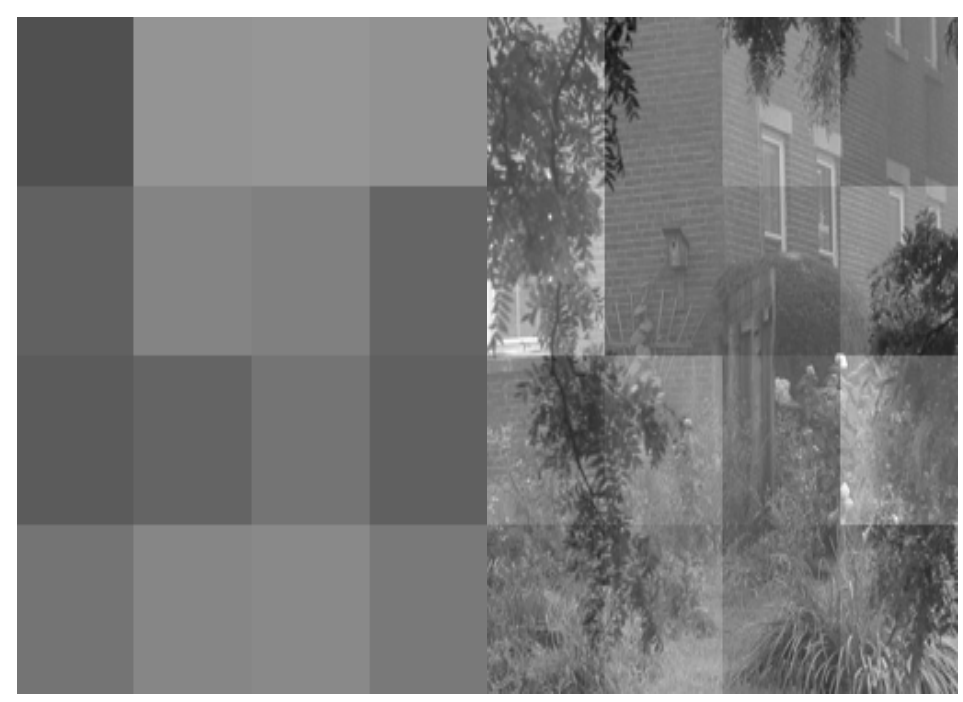

Figure 2 (a) Estimated Airlight map Restored Image before Smoothing



Figure 2 (b) Estimated Airlight map Restored Image after Smoothing 
International Journal of Information Sciences and Techniques (IJIST) Vol.2, No.3, May 2012

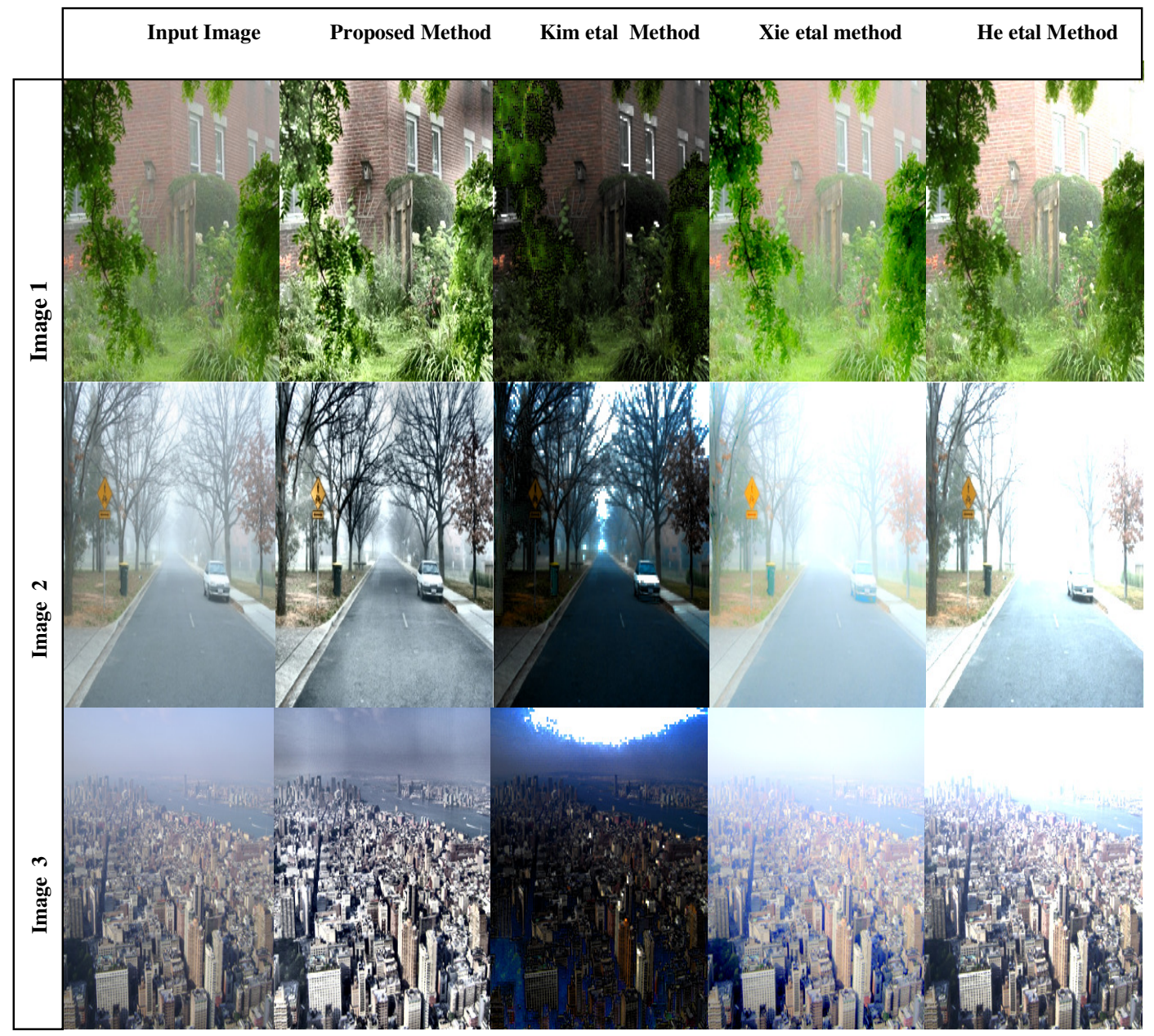

Figure 3 Hazy Images as Input and Comparison of Dehazed Images by various algorithms 


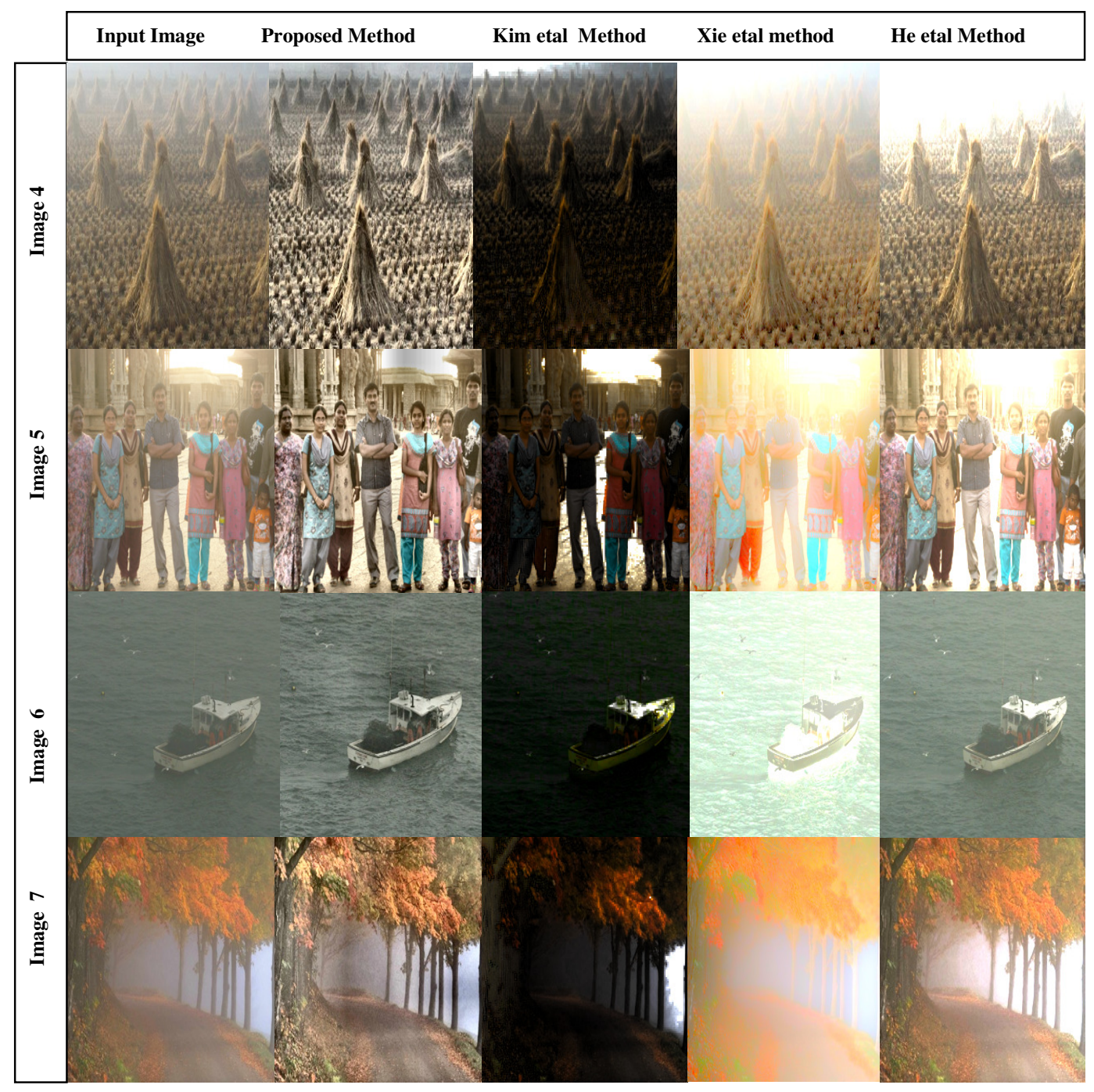

Figure 4 Hazy Images as Input and Comparison of Dehazed Images by various algorithms

Table 1 and Table 2 depicts the proposed algorithm has better performance having the highest tenengrad and contrast improvement index measures among other methods. Figure 3 and Figure 4 depicts various results of the algorithms wherein the other methods does not remove haze completely or degrade the image resulting in poor quality. The proposed method preserves the details of the image and also removes the haze efficiently. 
Table 1.Tenengrad values

\begin{tabular}{|c|c|c|c|c|c|c|}
\hline Image & $\begin{array}{c}\text { Original } \\
\text { image }\end{array}$ & $\begin{array}{c}\text { He etal } \\
\text { method }\end{array}$ & $\begin{array}{c}\text { Ding etal } \\
\text { method }\end{array}$ & $\begin{array}{c}\text { Xie etal } \\
\text { method }\end{array}$ & $\begin{array}{c}\text { Dehazing } \\
\text { using PSO }\end{array}$ & $\begin{array}{l}\text { Proposed } \\
\text { method } \\
\text { using ABC }\end{array}$ \\
\hline 1 & 1309831 & 92049 & 3031144 & 788740 & 7319721 & 52092818 \\
\hline 2 & 701286 & 30631 & 974296 & 12460000 & 8703646 & 46385527 \\
\hline 3 & 1582132 & 100053 & 3878440 & 1274500 & 14602661 & 54763393 \\
\hline 4 & 613077 & 107355 & 894088 & 547420 & 5707143 & 54094743 \\
\hline 5 & 659622 & 74909 & 1544002 & 5349100 & 8523587 & 53026664 \\
\hline 6 & 605532 & 64051 & 965403 & 431900 & 707074 & 15311663 \\
\hline 7 & 548885 & 66246 & 1479296 & 1249900 & 2819934 & 34701134 \\
\hline
\end{tabular}

Table 2.Contrast Improvement Index

\begin{tabular}{|c|c|c|c|c|c|c|}
\hline Image & $\begin{array}{c}\text { Original } \\
\text { image }\end{array}$ & $\begin{array}{c}\text { He etal } \\
\text { method }\end{array}$ & $\begin{array}{c}\text { Ding etal } \\
\text { method }\end{array}$ & $\begin{array}{c}\text { Xie etal } \\
\text { method }\end{array}$ & $\begin{array}{c}\text { Dehazing } \\
\text { using PSO }\end{array}$ & $\begin{array}{l}\text { Proposed } \\
\text { method } \\
\text { using ABC }\end{array}$ \\
\hline 1 & 39.3199 & 0.2630 & 53.2344 & 0.1164 & 40.9097 & 38.6072 \\
\hline 2 & 44.8933 & 0.2489 & 38.8303 & 45.1062 & 67.3529 & 68.4652 \\
\hline 3 & 28.904 & 0.2121 & 39.1314 & 11.4662 & 44.0511 & 44.2775 \\
\hline 4 & 36.3144 & 0.2358 & 33.8525 & 40.6342 & 44.3183 & 46.6907 \\
\hline 5 & 28.3586 & 0.1679 & 13.9295 & 48.5689 & 39.5221 & 45.3535 \\
\hline 6 & 8.9947 & 0.0485 & 17.3126 & 0.0469 & 24.2855 & 26.8489 \\
\hline 7 & 38.2544 & 0.1926 & 42.2278 & 0.0687 & 50.9545 & 52.2699 \\
\hline
\end{tabular}

\section{CONCLuSion}

Optimal airlight is estimated using $\mathrm{ABC}$ and dehazed image is restored by a novel technique based on the human visual modelwhich has been proposed in this paper.Artificial Bee Colony Optimisation performs better than Particle Swarm Optimisation in dehazing and the contrast measures are found to be increased. The performance of the proposed work is validated quantitatively with contrast parameter Tenengrad and contrast improvement index.

\section{REFERENCES}

[1] Y. S. Zhai and X. M. Liu, "An improved fog- degraded image enhancement algorithm," Wavelet Analysis and Pattern Recognition.

[2] S. G. Narasimhan and S. K. Nayar, "Contrast restoration of weather degraded images," IEEE Transactions on Pattern Analysis and Machine Intelligence, vol. 25, pp. 713-724, 2003

[3] E. Namer and Y. Y. Schechner, "Advanced visibility improvement based on polarization filtered images," Proc. SPIE, vol. 5888, pp.36-45, 2005.

[4] Y. Y. Schechner, S. G. Narasimhan, and S. K. Nayar, "Polarization-based vision through haze," Applied Optics, vol. 42,pp.511-525, 2003.

[5] J. P. Oakley and H. Bu, "Correction of Simple Contrast Loss in Color images," Image Processing,IEEE Transactions on, vol. 16, pp. 511-522, 2007.

[6] Y. Yitzhaky, I. Dror, and N. S. Kopeika, "Restoration of atmospherically blurred images accordingto weather-predicted atmospheric modulation transfer functions," Optical Engineering, vol 36,p.3064,1997. 
[7] K. K. Tan and J. P. Oakley, "Physics-based approach to color ," image enhancement in poor visibility conditions of the Optical Society of merica A, vol. 18, pp. 2460-2467, 2001

[8] R. S. Sirohi, "Effect of fog on the color of a distant light source," Journal of Physics D Applied Physics, vol. 3, pp. 96-99, 1970.

[9] S. Shwartz, E. Namer, and Y. Y. Schechner, "Blind haze separation" International Conference on Computer Vision and pattern recognition,2006.

[10] Y. Yitzhaky, I. Dror, and N. S. Kopeika, "Restoration of atmospherically blurred images according to weather-predicted atmospheric modulation transfer functions," Optical Engineering,vol36,p,3064,1997.

[11] K. K. Tan and J. P. Oakley, "Physics-based approach to color Image enhancement in poor visibility conditions," Journal of the Optical Society of America A, vol. 18, pp. 2460-2467, 2001.

[12] R. S. Sirohi, "Effect of fog on the colour of a distant light source," Journal of Physics D Applied Physics, vol. 3, pp. 96-99, 1970.

[13] S. Shwartz, E. Namer, and Y. Y. Schechner, "Blind haze separation," International Conference on Computer Vision and PatternRecognition,2006.

[14] Kaiming HeJian SunXiaoou Tang“ Single Image Haze Removal Using Dark Channel Prior"Computer Vision and Pattern Recognition, 2009. CVPR 2009.

[15] Ding Xiao Jun Ohya "Contrast Enhancement Of Color Images Based On Wavelet Transform AndHuman Visual System “ ,Proceedings of the IASTED International Conference in Graphics and Visualisation Engineering, January 3-5 ,2007.

[16] Bin Xie, Fan Gua Improved Single Image Dehazing Using Dark Channel Prior and Multi-scale Retinex “,2010 International Conference on Intelligent System Design and Engineering Application.

[17] S.MohamedMansoorRoomi,R.Bhargavi,S.Bhumesh "Particle Swarm Optimisation Based Image Dehazing",2012 National Conference on Signal and Image Processing.

\section{Authors}

S.Mohamed Mansoor Roomi received his B.E degree in Electronics and communication Engineering from Madurai Kamarajar University, in 1990.and the M.E(Power Systems)\&ME (Communication Systems) from Thiagarajar College of Engineering, Madurai in 1992\&1997 and $\mathrm{PhD}$ in 2009 from Madurai Kamarajar University . His primary Research Interests include Image Enhancement and Analysis.

R.Bhargaviis currently doing her under graduation in Electronics and Communication Engineering at Thiagarajar College of Engineering, Madurai,India. Her research interests are in the area of Image Enhancement and Image Analysis.

S.Bhumesh is currently doing his under graduation in Electronics and Communication Engineering at Thiagarajar College of Engineering, Madurai,India. His research interests are in the area of Image Enhancement and Image Analysis.
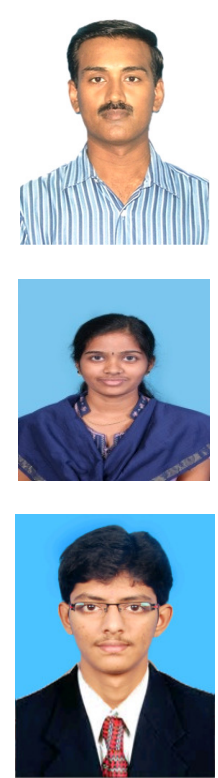